\title{
Numerical study of electrostatic electron cyclotron harmonic waves due to Maxwellian ring velocity distributions
}

\author{
Takayuki Umeda \\ Solar-Terrestrial Environment Laboratory, Nagoya University, Nagoya, Aichi 464-8601, Japan \\ (Received August 3, 2007; Revised September 18, 2007; Accepted October 2, 2007; Online published November 30, 2007)
}

\begin{abstract}
Excitation of electrostatic electron cyclotron harmonic $(\mathrm{ECH})$ waves is studied by performing linear dispersion analysis and particle-in-cell computer simulation. The ECH wave emissions can be excited by a positive slope in a velocity distribution function perpendicular to the ambient magnetic field, such as that due to a loss cone or ring velocity distribution. However, there exists no analytic expression for integration of Maxwellian ring velocity distribution functions. Here we present a method to solve the linear dispersion relations of Maxwellian ring velocity distribution functions with numerical integration. The obtained dispersion relations are confirmed by particle-in-cell simulation.
\end{abstract}

Key words: Electron cyclotron waves, linear dispersion analysis, numerical simulation.

\section{Introduction}

Electrostatic electron cyclotron harmonic $(\mathrm{ECH})$ waves are observed in the near-Earth equatorial region (e.g., Kennel et al., 1970; Rönnmark et al., 1978; Kurth et al., 1979a, b, 1980; Rönnmark and Christiansen, 1981; Horne et al., 1981, 1987; Koons and Fennel, 1984; Matsumoto and Usui, 1997; Usui et al., 1997, 1999a, b; Shinbori et al., 2007). The ECH wave emissions were observed at frequencies $f \sim\left(n+\frac{1}{2}\right) f_{c e}$, where $n$ is a positive integer and $f_{c e}$ denotes the electron cyclotron frequency.

It is well known that the ECH waves can be excited by an electrostatic instability induced when an electron velocity distribution function $F\left(v_{\|}, v_{\perp}\right)$ (where $v_{\|}$and $v_{\perp}$ are velocity components parallel and perpendicular to the ambient magnetic field, respectively) has a region of positive gradient, i.e., $\partial F / \partial v_{\perp}>0$. Thus, a loss cone or ring velocity distribution is likely to drive ECH waves unstable, and the presence of the cold background electrons strongly affects the growth rates (Fredricks, 1971; Young et al., 1973; Young, 1975; Ashour-Abdalla et al., 1975, 1979; AshourAbdalla and Kennel, 1978a, b), which may explain the enhancement of such waves near the plasmapause.

The effects of a loss cone distribution have been examined in a number of theoretical linear dispersion analyses of ECH waves whereby the loss cone feature of the distribution was modeled using subtracted Maxwellian velocity distribution functions (e.g., Ashour-Abdalla et al., 1975, 1979; Ashour-Abdalla and Kennel, 1978a, b). However, selfconsistent particle-in-cell (PIC) simulations of loss cone distributions, which can be used to analyze nonlinear effects, are difficult to be carried out. Since a loss cone distribution, which is relatively narrow, as expected from the

Copyright (c) The Society of Geomagnetism and Earth, Planetary and Space Sciences (SGEPSS); The Seismological Society of Japan; The Volcanological Society of Japan; The Geodetic Society of Japan; The Japanese Society for Planetary Sciences; TERRAPUB magnetospheric conditions in which these waves are observed ( $\sim$ few degrees), would be weakly unstable, it is difficult to study with PIC codes because of its high numerical noise level.

For the reason, the excitation of ECH waves has been examined by means of two-dimensional PIC simulations assuming a Maxwellian ring distribution (Ashour-Abdalla et al., 1980; Umeda et al., 2007). The Maxwellian ring velocity distribution was adopted since the resulting physical processes are similar to those occurring in a loss cone distribution. In contrast, the electrostatic linear dispersion relation for Maxwellian ring velocity distributions has not been analyzed because there is no analytic expression for the integral of ring distribution functions over $v_{\perp}$. Thus, cold plasma approximations, i.e., the Dirac delta functions, were used in previous theoretical works on the linear dispersion relation of ring velocity distributions (e.g., Fredricks, 1971; Tataronis and Crawford, 1970a, b; Lee and Birdsall, 1979; Sotnikov et al., 1995; Convery, 1997). The purpose of this paper is to present a method for solving the linear dispersion relations of Maxwellian ring velocity distributions with a numerical integration. The results of the linear dispersion analysis are compared against PIC simulation results.

The paper is structured as follows. The Maxwellian ring velocity distribution is introduced and its linear dispersion equation is derived in Section 2. The linear dispersion relation of electrostatic ECH waves is analyzed and compared against PIC simulation in Section 3. A summary and discussion of the results are presented in Section 4.

\section{Theoretical Formulation}

The starting point of the present analysis is the Harris dispersion equation of electrostatic waves in a uniform magnetic field (Harris, 1959), 


$$
\begin{aligned}
0= & D\left(\omega^{*}, k_{\|}, k_{\perp}\right) \\
= & 1-\sum_{s} \frac{\Pi_{s}^{2}}{k_{\|}^{2}+k_{\perp}^{2}} \sum_{n} \int_{-\infty}^{\infty} \int_{0}^{\infty} \frac{J_{n}^{2}\left(\frac{k_{\perp} v_{\perp}}{\Omega_{s}}\right)}{k_{\|} v_{\|}-\omega^{*}+n \Omega_{s}} \\
& \cdot\left(k_{\|} \frac{\partial F_{s}}{\partial v_{\|}}+\frac{n \Omega_{s}}{v_{\perp}} \frac{\partial F_{s}}{\partial v_{\perp}}\right) 2 \pi v_{\perp} \mathrm{d} v_{\perp} \mathrm{d} v_{\|},
\end{aligned}
$$

where $\Pi_{s}=\frac{q_{s}^{2} n_{s}}{\epsilon_{0} m_{s}}$ and $\Omega_{s}=\frac{q_{s}}{m_{s}} B_{0}$ represent the plasma frequency and the cyclotron frequency, respectively, for species $s, \omega^{*} \equiv \omega+i \gamma$ represents the complex frequency, and $k_{\|}, k_{\perp}$, and $v_{\|}, v_{\perp}$ represent wavenumbers and velocities in the directions parallel and perpendicular to the ambient magnetic field $B_{0}$, respectively.

We use a Maxwellian ring velocity distribution function,

$$
\begin{gathered}
F_{s}\left(v_{\|}, v_{\perp}\right)=F_{s \|}\left(v_{\|}\right) F_{s \perp}\left(v_{\perp}\right), \\
F_{s \|}\left(v_{\|}\right)=\frac{1}{\sqrt{2 \pi} V_{t s \|}} \exp \left[-\frac{v_{\|}^{2}}{2 V_{t s \|}^{2}}\right], \\
F_{s \perp}\left(v_{\perp}\right)=\frac{1}{2 \pi V_{t s \perp}^{2} A_{s}} \exp \left[-\frac{\left(v_{\perp}-V_{r s}\right)^{2}}{2 V_{t s \perp}^{2}}\right], \\
A_{s}=\exp \left[-\frac{V_{r s}^{2}}{2 V_{t s \perp}^{2}}\right]+\frac{\sqrt{\pi} V_{r s}}{\sqrt{2} V_{t s \perp}} \operatorname{erfc}\left[-\frac{V_{r s}}{\sqrt{2} V_{t s \perp}}\right],
\end{gathered}
$$

where $V_{r}, V_{t||}$, and $V_{t \perp}$ are the ring velocity and thermal velocities in the direction parallel and perpendicular to the ambient magnetic field, respectively. The velocity component parallel to the ambient magnetic field $v_{\|}$is defined from $-\infty$ to $\infty$, while the perpendicular velocity $v_{\perp}$ is defined from 0 to $\infty$.

The dispersion equation is derived by inserting Eq. (2) into Eq. (1). The dispersion equation contains the following four integrals:

$$
\begin{gathered}
\int_{-\infty}^{\infty} \frac{k_{\|}}{k_{\|} v_{\|}-\omega^{*}+n \Omega_{s}} \frac{\partial F_{s \|}}{\partial v_{\|}} \mathrm{d} v_{\|}=\frac{1}{2 V_{t s \|}^{2}} Z^{\prime}\left(\zeta_{s}\right), \\
\int_{-\infty}^{\infty} \frac{F_{s \|}}{k_{\|} v_{\|}-\omega^{*}+n \Omega_{s}} \mathrm{~d} v_{\|}=\frac{1}{\sqrt{2} k_{\|} V_{t s \|}} Z\left(\zeta_{s}\right), \\
\int_{0}^{\infty} J_{n}^{2}\left(\frac{k_{\perp} v_{\perp}}{\Omega_{s}}\right) F_{s \perp} 2 \pi v_{\perp} \mathrm{d} v_{\perp}=\frac{2}{A_{s}} X_{n}\left(a_{s}, b_{s}\right), \\
\int_{0}^{\infty} J_{n}^{2}\left(\frac{k_{\perp} v_{\perp}}{\Omega_{s}}\right) \frac{n \Omega_{s}}{v_{\perp}} \frac{\partial F_{s \perp}}{\partial v_{\perp}} 2 \pi v_{\perp} \mathrm{d} v_{\perp} \\
=-\frac{2 n \Omega_{s}}{V_{t s \perp}^{2} A_{s}} Y_{n}\left(a_{s}, b_{s}\right),
\end{gathered}
$$

where

$$
\zeta_{s}=\frac{\omega^{*}-n \Omega_{s}}{\sqrt{2} k_{\|} V_{t s \|}}, \quad a_{s}=\frac{\sqrt{2} k_{\perp} V_{t s \perp}}{\Omega_{s}}, \quad b_{s}=\frac{V_{r s}}{\sqrt{2} V_{t s \perp}} .
$$

Here the left-hand side of Eqs. (3) and (4) are analytically expressed by using the plasma dispersion function $Z(\zeta)$. For the left-hand side of Eqs. (5) and (6), on the other hand, the following two new functions are introduced,

$$
\begin{aligned}
& X_{n}(a, b) \equiv \int_{0}^{\infty} J_{n}^{2}(a y) \exp \left[-(y-b)^{2}\right] y \mathrm{~d} y, \\
& Y_{n}(a, b) \equiv \int_{0}^{\infty} J_{n}^{2}(a y) \exp \left[-(y-b)^{2}\right](y-b) \mathrm{d} y .
\end{aligned}
$$
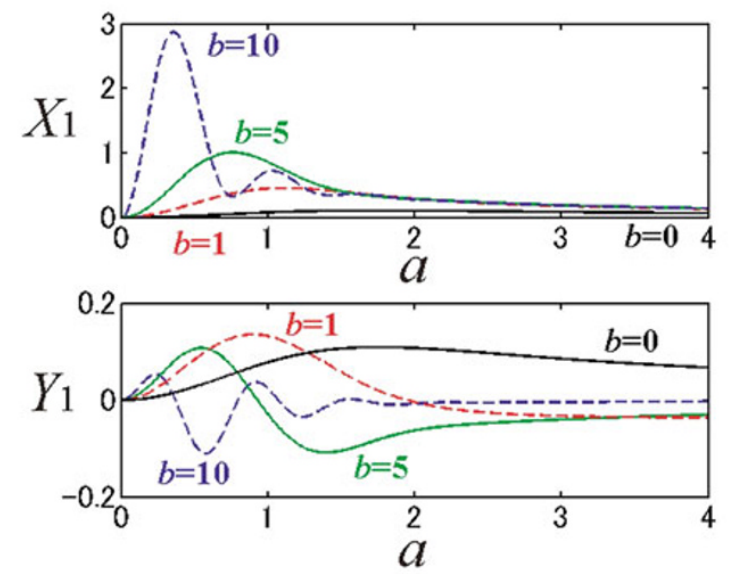

Fig. 1. Plots of the functions $X_{n}$ and $Y_{n}$ for $n=1$.

It is well known that there are no analytic expressions of these two integrals except for $b=0$,

$$
X_{n}(a, 0)=Y_{n}(a, 0)=\frac{1}{2} \exp \left(-\frac{a^{2}}{2}\right) I_{n}\left(\frac{a^{2}}{2}\right) .
$$

Thus, here we compute the values of the functions $X_{n}$ and $Y_{n}$ using numerical integration. The linear dispersion relation of electrostatic waves in Maxwellian ring velocity distributions is then obtained,

$$
\begin{aligned}
0= & D\left(\omega^{*}, k_{\|}, k_{\perp}\right) \\
= & 1-\sum_{s} \frac{\Pi_{s}^{2}}{\left(k_{\|}^{2}+k_{\perp}^{2}\right) V_{t s \|}^{2} A_{s \perp}} \\
& \cdot \sum_{n}\left[X_{n}\left(a_{s}, b_{s}\right) Z^{\prime}\left(\zeta_{s}\right)-\frac{\sqrt{2} n \Omega_{s} V_{t s \|}}{k_{\|} V_{t s \perp}^{2}} Y_{n}\left(a_{s}, b_{s}\right) Z\left(\zeta_{s}\right)\right]
\end{aligned}
$$

In this study, the interval [ $\left.\max \left(0, b_{s}-5\right), b_{s}+5\right]$ is broken into 100 pieces and Simpson's rule is used for numerical integration of the functions (7) and (8). Plots of functions $X_{n}$ and $Y_{n}$ for $n=1$ are shown in Fig. 1. We do not need to integrate over the full interval $[0, \infty]$ because $e^{-5^{2}}(\sim$ $\left.10^{-11}\right)$ is small enough. It is also noted that numerical error between numerical integrals with 100 pieces and that with 1000 pieces is $\sim 10^{-8}$.

\section{Numerical Results}

A basic plasma model is used here for the ECH instability (Young et al., 1973) in which an unstable plasma is composed of a hot free energy source and a cold component. Thus, the subscript $s=h$ and $c$ represent hot and cold electrons, respectively. The free energy source consists of a ring in velocity space in the direction perpendicular to the ambient magnetic field. The hot ring Maxwellian electrons have the ring velocity $V_{r h}=V_{r}$, whereas the cold background electrons have zero ring velocity $\left(V_{r c}=0\right)$. Isotropic thermal plasmas are also assumed, i.e., $V_{t s \|}=V_{t s \perp}=V_{t s}$. Since only high-frequency electrostatic electron plasma waves with a normalized frequency $\omega /\left|\Omega_{e}\right| \sim 1.5$, are to be considered here, the ions are assumed to be an immobile background.

It is assumed that the total electron plasma frequency $\Pi_{e} \equiv \sqrt{\Pi_{h}^{2}+\Pi_{c}^{2}}=1.0$, the electron cyclotron frequency 

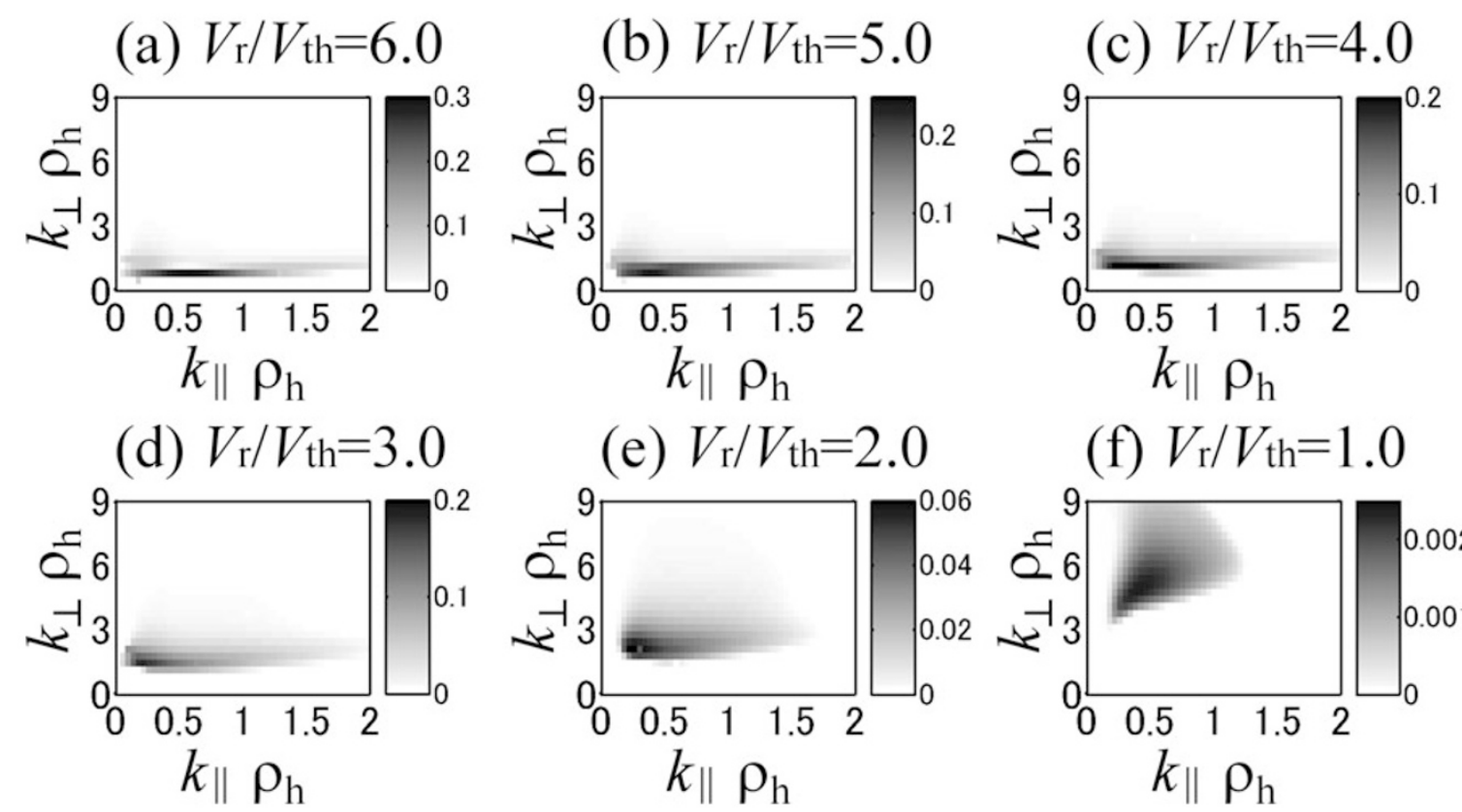

(f) $V_{\mathrm{r}} / V_{\mathrm{th}}=1.0$

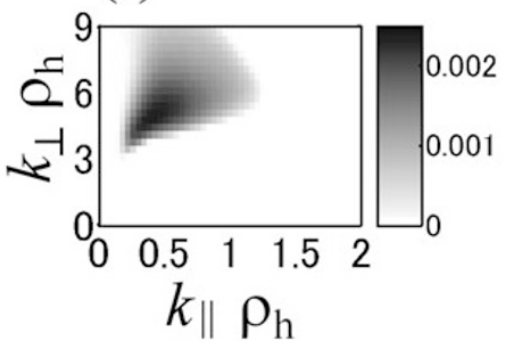

Fig. 2. Growth rates of electrostatic ECH waves in $\left(k_{\|}, k_{\perp}\right)$ space with $V_{t c} / V_{t h}=0.1, V_{r} / V_{t h}=$ (a) 6.0, (b) 5.0, (c) 4.0, (d) 3.0, (e) 2.0, and (f) 1.0.

$\Omega_{e}\left(=\Omega_{h}=\Omega_{c}\right)=-0.3$, the density ratio $n_{h} / n_{c}=4.0$, and the isotropic thermal velocity of hot ring electrons $V_{t h}=1.0$. It is also assumed that a condition outside the plasmapause where a warm plasma with a positive slope in the velocity distribution function is injected into a plasmaspheric low-density cold plasma on closed field lines.

\subsection{Growth rates of ECH waves in $\left(k_{\|}, k_{\perp}\right)$ space}

Figures 2 and 3 show the normalized growth rates of ECH waves $\gamma /\left|\Omega_{e}\right|$ in $\left(k_{\|}, k_{\perp}\right)$ space. In Fig. 2 , the ring velocity is varied as $V_{r}=6.0,5.0,4.0,3.0,2.0$, and 1.0 with a constant isotropic thermal velocity of cold electrons $V_{t c}=$ 0.1. In Fig. 3, the thermal velocity of cold electrons is varied as $V_{t c}=0.2,0.3$, and 0.4 with a constant ring velocity $V_{r}=3.0$. The parallel and perpendicular wavenumbers $k_{\|}$ and $k_{\perp}$ are normalized by the gyro-radius of hot electrons $\rho_{h} \equiv V_{t h} /\left|\Omega_{e}\right|$. In the present $\left(k_{\|}, k_{\perp}\right)$ space search, we used Muller's complex-root finder (Muller, 1956) to obtain the numerical solution to the dispersion equation (Eq. (9)).

The linear dispersion analysis shows that the ECH waves have positive growth rates at the frequency range of $\omega /\left|\Omega_{e}\right|=1.4 \sim 1.8$, which is below the upperhybrid resonance frequency of the cold background electrons, $\sqrt{\Pi_{c}^{2}+\Omega_{e}^{2}}$. Thus, the cold background electrons affects the linear dispersion relation of the ECH waves (Fredricks, 1971; Young et al., 1973; Young, 1975; AshourAbdalla et al., 1975, 1979; Ashour-Abdalla and Kennel, 1978a, b).

The ECH waves have maximum growth rates at a parallel wavenumber range of $k_{\|} \rho_{h}=0.25 \sim 0.5$. A typical cyclotron resonance velocity is given by

$$
V_{p \|}=\frac{\omega+\Omega_{e}}{k_{\|}} \sim \frac{0.7\left|\Omega_{e}\right|}{0.3 / \rho_{h}}=2.33 V_{t h} .
$$

The ECH waves have positive growth rates in a resonance velocity range faster than the thermal velocity of hot ring electrons. Thus, the growth of the ECH waves is not af- fected by the Landau damping.

Figure 2 shows that the perpendicular wavenumber of the $\mathrm{ECH}$ mode becomes larger as the ring velocity becomes slower. The ECH waves have maximum growth rates at $k_{\perp} V_{r} / \Omega_{e} \sim 5$ when the ring velocity is faster than the perpendicular thermal velocity of hot electrons. This result indicates that the perpendicular wavenumber of the $\mathrm{ECH}$ mode is determined by the ring velocity.

Figure 3 shows that the growth rate of $\mathrm{ECH}$ mode becomes lower as the thermal velocity of cold electrons becomes faster. The parallel wavenumber range of the unstable ECH waves becomes narrower as the thermal velocity of cold electrons becomes faster. The perpendicular wavenumber range of the unstable $\mathrm{ECH}$ waves is also slightly modified. The wave normal angle of the most unstable ECH mode is modified by the cold electron temperature. To study the effect of both Landau and cyclotron damping, results with $V_{t c \|}=0.4, V_{t c \perp}=0.1$ and $V_{t c \|}=0.1, V_{t c \perp}=0.4$ are shown in Fig. 3(d) and Fig. 3(e), respectively. When the parallel thermal velocity of cold electrons increases, the parallel wavenumber range of $\mathrm{ECH}$ waves becomes narrower while the perpendicular wavenumber range does not change (see Fig. 2(d) and Fig. 3(d)). This results from the parallel thermal velocity affecting only $Z^{\prime}(\zeta)(=-2[1+\zeta Z(\zeta)])$ in Eq. (9). On the other hand, when the perpendicular thermal velocity of the cold electrons increases, the growth rate of $\mathrm{ECH}$ mode decreases and the perpendicular wavenumber range becomes slightly narrower. The perpendicular thermal velocity affects the arguments of both $X_{n}$ and $Y_{n}$ in Eq. (9). A larger $V_{t \perp}$ gives a larger $a$ and a smaller $b$, which can change the properties of $X_{n}$ and $Y_{n}$, as shown in Fig. 1, and the cyclotron resonance condition can be modified. In summary, the parallel thermal velocity determines the parallel wavenumber range of ECH waves while the perpendicular thermal velocity determines the dispersion relation 
(a) $\mathrm{V}_{\mathrm{tc}} / \mathrm{V}_{\mathrm{th}}=0.2$

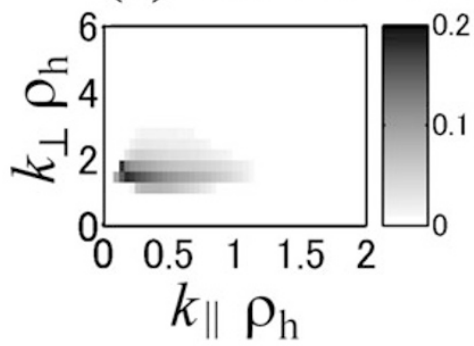

(d) $\mathrm{V}_{\text {tctl }} / \mathrm{V}_{\text {th }}=0.4$

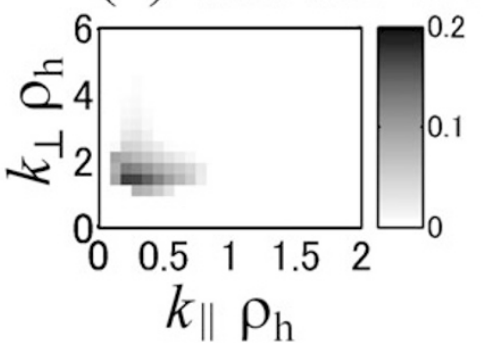

(b) $\mathrm{V}_{\mathrm{tc}} / \mathrm{V}_{\mathrm{th}}=0.3$

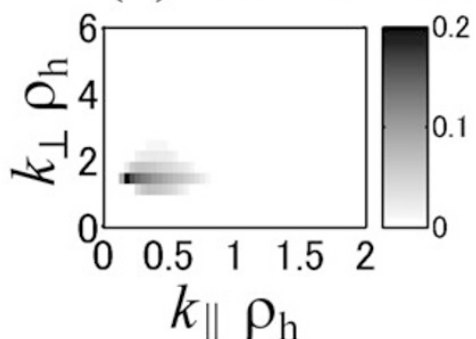

(e) $\mathrm{V}_{\text {tc } \perp} / \mathrm{V}_{\text {th }}=0.4$

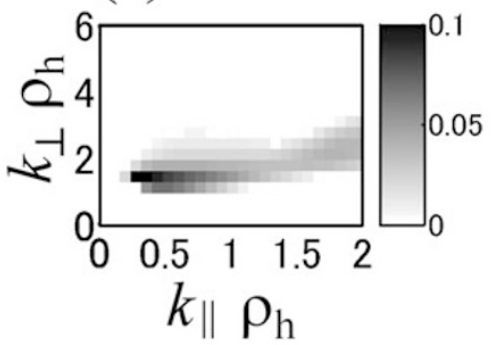

(c) $\mathrm{V}_{\text {tc }} / \mathrm{V}_{\text {th }}=0.4$

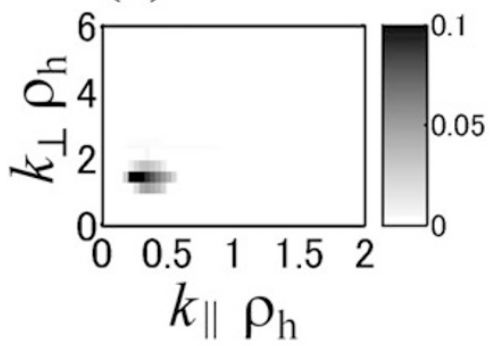

Fig. 3. Growth rates of electrostatic ECH waves in $\left(k_{\|}, k_{\perp}\right)$ space with $V_{r} / V_{t h}=3.0, V_{t c} / V_{t h}=$ (a) 0.2 , (b) 0.3 , and (c) 0.4 .

and growth rate of ECH waves.

\subsection{Comparison with PIC simulation}

The present linear analysis was confirmed by performing a PIC simulation. A 2.5-dimensional electromagnetic PIC code (Umeda, 2004) is used in which Maxwell's equations and the equations of motion for individual charged particles are solved in a self-consistent manner in two spatial and three velocity dimensions. The simulation system is taken in the $x-y$ plane with a simulation box size of $L_{x} \times L_{y}=512 \lambda_{e} \times 128 \lambda_{e}$, where $\lambda_{e}=V_{t h} / \Pi_{e}$ is the electron Debye length. The ambient magnetic field is taken in the $x$ direction. Periodic boundary conditions are imposed in both the $x$ and $y$ directions for both fields and particles. A total of 256 particles were used per cell for each component. The time step and the grid spacing of the present simulation are $\Pi_{e} \Delta t=0.032$ and $\Delta x=\Delta y=\lambda_{e}$, respectively. Detained descriptions on the simulation setup are given by Umeda et al. (2007).

The maximum growth rates of the $\mathrm{ECH}$ waves obtained by the PIC simulation and the numerical dispersion analysis are compared in Fig. 4. The bracketed numbers represent mode numbers of the most unstable ECH mode in each PIC simulation, where the mode number corresponds to the number of spatial oscillation cycles in the $x$ and $y$ directions, i.e., $\left(L_{x} k_{\|} / 2 \pi, L_{y} k_{\perp} / 2 \pi\right)$. Thus, the maximum growth rate is also a function of wavenumbers. Note that the wavenumbers and the maximum growth rates do not necessarily correspond to the theoretical analysis shown in Figs. 2 and 3 because of the discrete wavenumbers in computer simulations.

Figure 4(a) shows the simulated and theoretical growth rates of as a function of ring velocity $V_{r}$, and Fig. 3(b) shows the growth rates of ECH waves as a function of cold thermal velocity $V_{t c}$. The result shows an excellent agreement between the PIC simulation and the numerical analysis of the linear dispersion relation (Eq. (9)). Note (a)
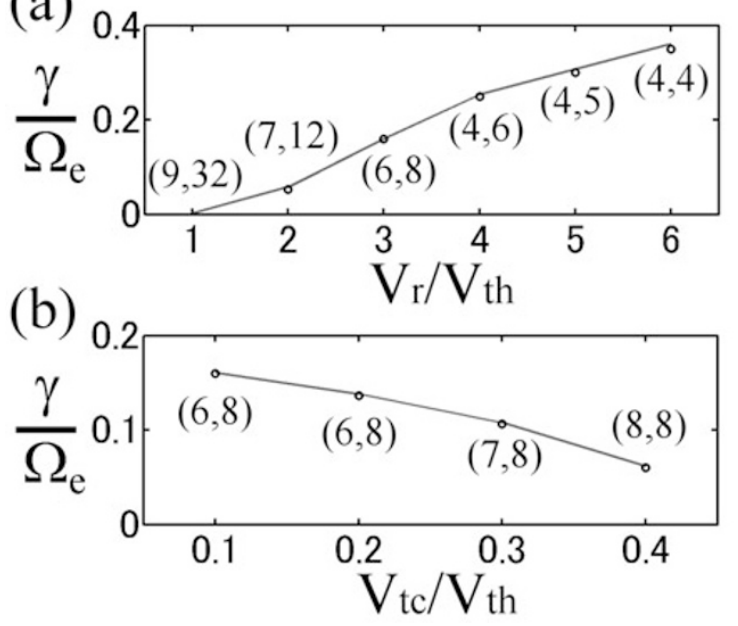

Fig. 4. Comparison between PIC simulation and numerical analysis of the theoretical dispersion relations: Simulated and theoretical maximum growth rates of ECH waves as functions of (a) $V_{r}$ and (b) $V_{t c}$. The theoretical growth rates are plotted as solid lines, while the simulated growth rates are plotted as circles. The numbers in parenthesis represent mode numbers of the most unstable ECH modes in the PIC simulation, $\left(L_{x} k_{\|} / 2 \pi, L_{y} k_{\perp} / 2 \pi\right)$ with $L_{x}=512 \lambda_{e}, L_{y}=128 \lambda_{e}$.

that we cannot find the growth of the $\mathrm{ECH}$ mode in the run with $V_{r} / V_{t h}=1.0$ in Fig. 4(a) because the growth rate is too small to follow a PIC simulation with a high numerical noise level.

\section{Summary and Discussion}

In the present paper, excitation of electrostatic electron cyclotron harmonic $(\mathrm{ECH})$ waves is studied by means of theoretical dispersion analysis and particle-in-cell (PIC) computer simulation. A theoretical expression for the $\mathrm{ECH}$ waves due to Maxwellian ring velocity distributions is first 
derived, and then the linear dispersion equation is numerically solved.

As there is no analytic expression for integrals of the Maxwellian ring velocity distribution over the velocity component perpendicular to the ambient magnetic field, the former was analyzed using the cold approximation (e.g., Fredricks, 1971; Tataronis and Crawford, 1970a, b; Lee and Birdsall, 1979; Sotnikov et al., 1995; Convery, 1997). In this study, the ring distribution is integrated numerically and dispersion relations are then derived. The obtained dispersion relations have been compared against the PIC simulations. The result shows an excellent agreement between the numerical analysis and the computer simulation.

In this study, we assumed a condition outside the plasmapause in which an unstable velocity distribution consists of a warm ring plasma and a plasmaspheric low-density cold plasma. Detained nonlinear consequences due to the ring velocity distribution have been reported by Umeda et al. (2007). Here, the perpendicular wavenumber of the ECH mode is shown to be proportional to the ring velocity when the ring velocity is faster than the thermal velocity of hot electrons. The parallel phase (resonant) velocity of the ECH mode becomes slightly faster as the ring velocity becomes faster. The temperature of the cold electrons affects the linear dispersion relation and the growth rate. Since the frequency of the ECH mode is determined by the upper-hybrid resonance frequency of cold electrons, the density ratio of cold-to-hot electrons is an important parameter. Thus, properties of ECH waves inside the plasmapause and plasmasphere would be different from those outside the plasmapause.

The present approach is useful when the perpendicular velocity distribution is not an analytic function. The integral can be easily obtained using Simpson's rule, and linear dispersion relations can be numerically derived in Eq. (9) as long as a parallel velocity distribution is a Maxwellian. When a parallel velocity distribution is not a Maxwellian nor an analytic function, however, it is not easy to integrate the parallel velocity distribution because the integral becomes a complex number. In such a case, a numerical method proposed by Nakamura and Hoshino (1998) would be useful to numerically derive linear dispersion relations.

Acknowledgments. The author is grateful to M. Ashour-Abdalla, D. Schriver, and R. L. Richard for discussions. The computer simulations were performed on the DataStar supercomputer system at the San Diego Supercomputer Center (SDSC) and the Columbia supercomputer system at the NASA Advanced Supercomputing (NAS) Division.

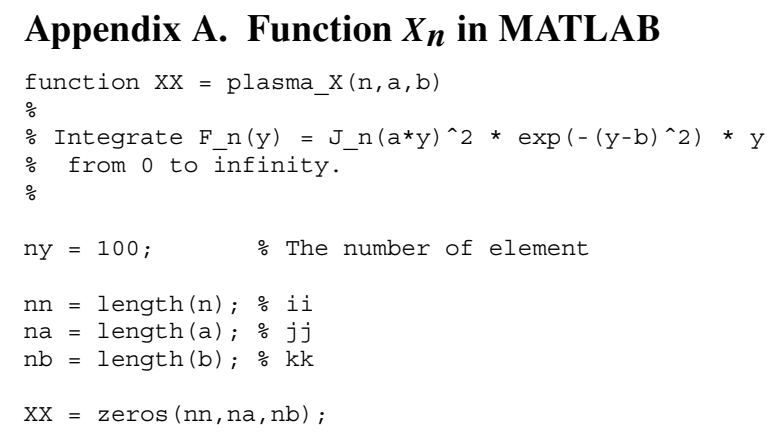

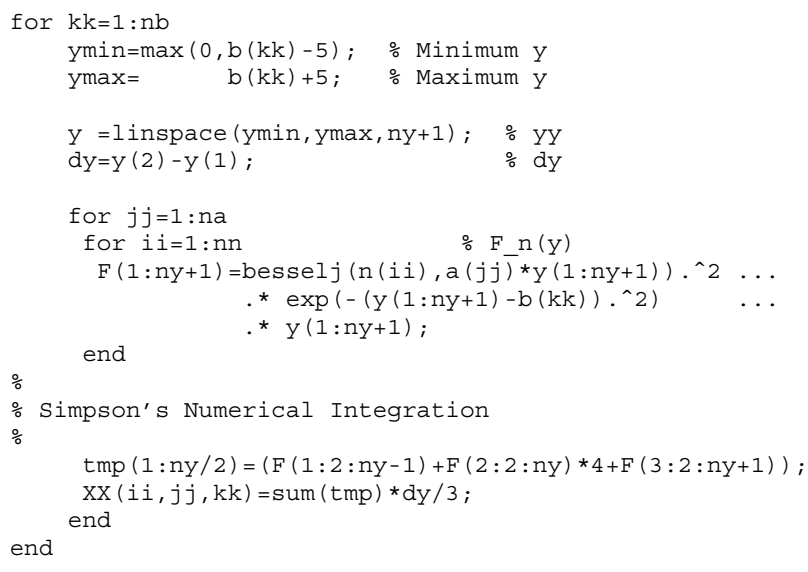

\section{Appendix B. Function $\boldsymbol{Y}_{\boldsymbol{n}}$ in MATLAB}

function YY $=$ plasma_Y $(n, a, b)$

Integrate $F \_n(y)=J \_n(a * y)^{\wedge} 2 * \exp \left(-(y-b)^{\wedge} 2\right) *(y-b)$ from 0 to infinity.

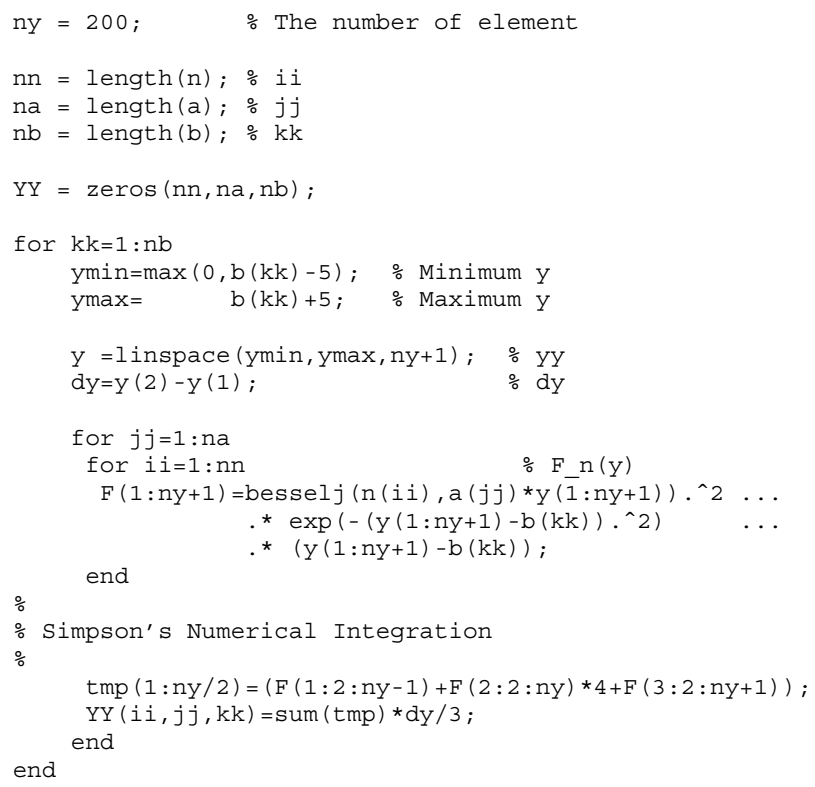

\section{References}

Ashour-Abdalla, M. and C. F. Kennel, Multi-harmonic electron cyclotron instabilities, Geophys. Res. Lett., 5, 711-714, 1978a.

Ashour-Abdalla, M. and C. F. Kennel, Nonconvective and convective electron cyclotron harmonic instabilities, J. Geophys. Res., 83, 1531-1543, 1978 b.

Ashour-Abdalla, M., G. Chanteur, and R. Pellat, A contribution to the theory of the electrostatic half-harmonic electron gyrofrequency waves in the magnetosphere, J. Geophys. Res., 80, 2775-2782, 1975.

Ashour-Abdalla, M., C. F. Kennel, and W. Livesey, A parametric study of electron multiharmonic instabilities in the magnetosphere, J. Geophys. Res., 84, 6540-6546, 1979.

Ashour-Abdalla, M., J.-N. Leboeuf, J. M. Dawson, and C. F. Kennel, A simulation study of cold electron heating by loss cone instabilities, Geophys. Res. Lett., 7, 889-892, 1980.

Convery, P., Ring and nongyrotropic distributions in the Earth's magnetosphere, Ph.D. Thesis, University of California, Los Angeles, USA, 1997.

Fredricks, R. W., Plasma instability at $(n+1 / 2) f c$ and its relationship to some satellite observations, J. Geophys. Res., 76, 5344-5348, 1971.

Harris, E. G., Unstable plasma oscillations in a magnetic field, Phys. Rew. Lett., 2, 34-36, 1959.

Horne, R. B., P. J. Christiansen, M. P. Gough, K. Rönnmark, J. F. E. Johnson, J. Sojka, and G. L. Wrenn, Amplitude variations of electron cyclotron harmonic waves, Nature, 294, 338-340, 1981. 
Horne, R. B., P. J. Christiansen, and M. P. Gough, Weak electrostatic waves near the upper hybrid frequency: A comparison between theory and experiment, J. Geophys. Res., 92, 3243-3259, 1987.

Kennel, C. F., F. L. Scarf, R. W. Fredricks, J. H. Mcghee, and F. V. Coroniti, VLF electric field observations in the inner magnetosphere, J. Geophys. Res., 75, 6136-6152, 1970.

Koons, H. C. and J. F. Fennel, Fine structure in electrostatic emission bands between electron gyrofrequency harmonics, J. Geophys. Res., 89, 30153018, 1984.

Kurth, W. S., M. Ashour-Abdalla, L. A. Frank, C. F. Kennel, D. A. Gurnett, D. D. Sentman, and B. G. Burek, A comparison of intense electrostatic waves near $\mathrm{f}_{\mathrm{UHR}}$ with linear instability theory, Geophys. Res. Lett., 6 , 487-490, 1979a.

Kurth, W. S., J. D. Craven, L. A. Frank, and D. A. Gurnett, Intense electrostatic waves near the upper hybrid resonance frequency, J. Geophys. Res., 84, 4145-4164, 1979b.

Kurth, W. S., L. A. Frank, D. A. Gurnett, B. G. Burek, and M. AshourAbdalla, Observations of a free-energy source for intense electrostatic waves, Geophys. Res. Lett., 7, 293-296, 1980.

Lee, J. K. and C. K. Birdsall, Velocity space ring-plasma instability, magnetized, Part I: Theory, Phys. Fluids, 22, 1306-1314, 1979.

Matsumoto, H. and H. Usui, Intense bursts of electron cyclotron harmonic waves near the dayside magnetopause observed by GEOTAIL, Geophys. Res. Lett., 24, 49-52, 1997.

Muller, D. E., A method for solving algebraic equations using an automatic computer, Math. Tables Aids Comput. (now Math. Comput.), 10, 208$215,1956$.

Nakamura, T. K. and M. Hoshino, One-over-polynomial approximation for linear kinetic dispersion and its application to relativistic cyclotron resonance, Phys. Plasmas, 5, 3547-3551, 1998.

Rönnmark, K. and P. J. Christiansen, Dayside electron cyclotron harmonic emissions, Nature, 294, 335-338, 1981.

Rönnmark, K., H. Borg, P. J. Christensen, M. P. Gough, and D. Jones, Banded electron cyclotron harmonic instability: A first comparison of theory and experiment, Space Sci.Rev., 22, 401-417, 1978.

Shinbori, A., T. Ono, M. Iizima, A. Kumamoto, S. Shirai, A. Hanaoka,
K. Okamoto, M. Ohashi, and H. Oya, Electrostatic electron cyclotron harmonic waves observed by the Akebono satellite near the equatorial region of the plasmasphere, Earth Planets Space, 59, 613-629, 2007.

Sotnikov, V. I., D. Schriver, M. Ashour-Abdalla, J. Ernstmeyer, and N Myers, Excitation of electron acoustic waves by a gyrating electron beam, J. Geophys. Res., 100, 19,765-19,772, 1995.

Tataronis, J. A. and F. Crawford, Cyclotron harmonic waves propagation and instabilities: I. Perpendicular propagation, J. Plasma Phys., 4, 231248, 1970a.

Tataronis, J. A. and F. Crawford, Cyclotron harmonic waves propagation and instabilities: II. Oblique propagation, J. Plasma Phys., 4, 249-264, $1970 b$.

Umeda, T., Study on nonlinear processes of electron beam instabilities via computer simulations, Ph.D. Thesis, Kyoto University, 2004.

Umeda, T., M. Ashour-Abdalla, D. Schriver, R. L. Richard, and F. V. Coroniti, Particle-in-cell simulation of Maxwellian ring velocity distribution, J. Geophys. Res., 112, A04212, doi:10.1029/2006JA012124, 2007.

Usui, H., J. Koizumi, and H. Matsumoto, Statistical study of electron cyclotron harmonic waves observed in the dayside magnetosphere, $A d v$ Space Res., 20, 857-860, 1997.

Usui, H., H. Matsumoto, T. Mukai, and Y. Saito, Geotail observation of electron cyclotron harmonic waves near the dayside magnetopause, $A d v$ Space Res., 24, 99-102, 1999a.

Usui, H., W. R. Paterson, H. Matsumoto, L. A. Frank, M. Nakamura, H Matsui, T. Yamamoto, O. Nishimura, and J. Koizumi, Geotail electron observations in association with intense bursts of electron cyclotron harmonic waves in the dayside magnetosphere, J. Geophys. Res., 104, 4477-4484, 1999b.

Young, T. S. T., Destabilization and wave-induced evolution of the magnetospheric plasma clouds, J. Geophys. Res., 80, 3995-4003, 1975.

Young, T. S. T., J. D. Callen, and J. E. McCune, High-frequency electrostatic waves in the magnetosphere, J. Geophys. Res., 78, 1082-1099, 1973

T. Umeda (e-mail: umeda@stelab.nagoya-u.ac.jp) 